WellBeing International

WBI Studies Repository

4-1994

\title{
Vocal Learning in Grey Parrots (Psittacus erithacus): Effects of Social Interaction, Reference, and Context
}

Irene M. Pepperberg

University of Arizona

Follow this and additional works at: https://www.wellbeingintlstudiesrepository.org/acwp_asie

Part of the Animal Studies Commons, Comparative Psychology Commons, and the Other Animal Sciences Commons

\section{Recommended Citation}

Pepperberg, I. M. (1994). Vocal learning in grey parrots (Psittacus erithacus): effects of social interaction, reference, and context. The Auk, 300-313.

This material is brought to you for free and open access by WellBeing International. It has been accepted for inclusion by an authorized administrator of the WBI Studies Repository. For more information, please contact wbisr-info@wellbeingintl.org.

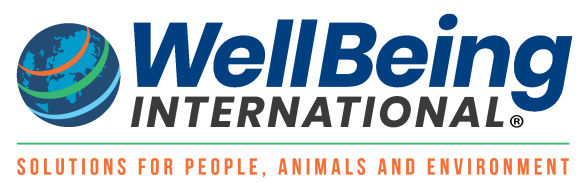




\title{
VOCAL LEARNING IN GREY PARROTS (PSITTACUS ERITHACUS): EFFECTS OF SOCIAL INTERACTION, REFERENCE, AND CONTEXT
}

\author{
IRENE M. PEPPERBERG \\ Department of Ecology and Evolutionary Biology, University of Arizona, \\ Tucson, Arizona 85721, USA
}

\begin{abstract}
For many passerines, the extent, timing, and even presence of allospecific vocal learning can be influenced by the form of input that is received. Little data exist, however, on vocal learning in parrots (Psittacidae). I have previously proposed that such vocal learning proceeds most readily when input is (1) referential, (2) contextually applicable, and (3) interactive. The referential aspect demonstrates the meaning of the code to be taught, the contextual aspect demonstrates the use that can be made of the information contained in the code, and the interactive aspect provides explicit training that is constantly adjusted to the level of the learner. To obtain information on the relative importance of these three aspects of input on learning in a mimetic species, I used three different conditions to train two juvenile Grey Parrots (Psittacus erithacus) to produce English labels to identify various common objects. Each bird experienced: (1) audiotaped tutoring, which was nonreferential, noninteractive, and did not demonstrate contextual applicability; (2) videotapes, which provided reference and limited information about context, but which were noninteractive; and (3) live human tutors, who interactively modeled the meaning and use of the labels to be learned. The birds learned only from the live tutors. A third parrot, trained on a separate set of labels by tutors who provided only limited reference and context for those vocalizations, learned to produce that set of labels without comprehension. The data suggest that, even for birds known for their mimetic abilities, social interaction, reference, and full contextual experience are important factors in learning to produce and comprehend an allospecific code. Received 22 April 1993, accepted 10 October 1993.
\end{abstract}

IN THE LAST DECADE, studies have shown how input affects allospecific avian vocal learning (review in Pepperberg 1991, 1993). For birds that favor conspecific learning (i.e. typically do not mimic other species in the field), allospecific learning is often affected by variation in social and environmental input. Such species (e.g. White-crowned Sparrows [Zonotrichia leucophrys], Baptista and Morton 1981, Baptista and Petrinovich 1984, 1986; Song Sparrows [Melospiza melodia], Marler and Peters 1977, 1987, Baptista 1988) may require visual and vocal interaction with a live tutor for complete allospecific song learning to occur. Less is known, however, about the effect of input on vocal learning in birds that are frequent mimics.

Data on vocal learning in some mimetic birds come from experiments on training these birds to reproduce human speech in the laboratory (review in Pepperberg 1988a). Little or nothing was learned by Indian Hill Mynahs (Gracula religiosa) exposed to tapes in social isolation (Grosslight et al. 1964, Grosslight and Zaynor 1967, Gossette 1969) or by an Indian Hill Mynah, Budgerigars (Melopsittacus undulatus), Blackbilled Magpies (Pica pica), a Yellow-headed Par- rot (Amazona ochrocephala), and a Grey Parrot (Psittacus erithacus) that experienced limited social input (Mowrer 1952, 1954, 1958). In contrast, Grey Parrots that received modeled, interactive human tutoring (Todt 1975, Pepperberg 1981, 1990a) acquired the targeted speech patterns. Given the reputed ease with which these mimetic birds are assumed to acquire any type of sound (see Amsler 1947), data on the effects of differential input were surprising. However, because different laboratories tested separate sets of conditions, the findings could have been a consequence of interlaboratory variation, as well as of the different learning conditions (Slater 1991).

The possible confounding factor of interlaboratory variation was countered in a single study on mimetic European Starlings (Sturnus vulgaris) that examined how competing forms of differential input affected learning (West et al. 1983). Seven birds, placed in three different groups, did or did not experience, in various combinations: human care and vocal interaction; tapes of human speech and whistles; and living quarters shared with Brown-headed Cowbirds ( $M o l-$ othrus ater) or other juvenile starlings. All birds 
learned vocalizations only from organisms with which they could interact (either human or cowbird) or learned sounds closely associated with the presence of such organisms (e.g. the opening of a door that preceded a morning greeting) and learned nothing from any noninteractive source. Such data demonstrated the effect of differential input on learning, but did not isolate which aspects of the input were critical for learning.

To demonstrate the relative importance of various aspects of input for learning, one must first identify the relevant aspects. According to a psychological construct called "social-modeling theory" (Bandura 1971, 1977), input can be characterized by three main aspects (Pepperberg 1985, 1988b, 1991, 1992a, Pepperberg and Neapolitan 1988, Pepperberg and SchinkeLlano 1991): (1) degree of referentiality, (2) scope of contextual applicability, and (3) extent of social interaction. Reference and contextual applicability refer to the real-world use of the input, and social interaction is a potent means of highlighting various components of the input. Reference is generally defined as the meaning of an utterance (e.g. the relationship between a label and the object to which it refers). Contextual applicability involves the particular situation in which an utterance is used and the effects of using the utterance. Social interaction acts to signal which components of the environment should be noted, emphasizes common attributes-and thus possible underlying rules-of diverse actions, and allows input to be continuously adjusted to the level of the learner. Interaction may also provide a contextual explanation of the reasons for the actions and demonstrate the consequences of the actions (for detailed discussion of these points, see Pepperberg 1993). Researchers can specifically design input that varies with respect to these aspects and then evaluate the relative effects of such variation.

To carry out such an evaluation, I designed experiments for one adult and two juvenile Grey Parrots. I examined how various forms of input affected the amount and type of their acquisition of an allospecific code, English speech. I studied how input might affect competence not only with respect to physical production, but also with respect to comprehension and appropriate use. In the experiment with the juveniles, I studied the relative effects of three types of input: (1) nonreferential, not contextually ap- plicable, and noninteractive input; (2) referential, minimally contextually applicable, and noninteractive input; and (3) referential, contextually applicable, and interactive input. In the experiment with the adult parrot, I studied the effect of input that was socially interactive but that minimized reference and contextual applicability. At the time, I chose not to study the effect of input that was referential, fully contextually applicable, and noninteractive because studies on other species suggested that such conditions lead at best to production without comprehension (see Savage-Rumbaugh et al. 1980a, b).

The results of these experiments not only provide information on Grey Parrots, but also suggest intriguing parallels between avian and human exceptional learning-learning that is unlikely in the normal course of development but that can occur under certain conditions (Pepperberg 1985, 1986, 1988a, 1993). Such behavior was first described by human social psychologists (Bandura 1971, 1977). I discuss the results in the context of my previous proposal that avian acquisition of an allospecific code is a particular form of exceptional learning; I suggest possible parallels between such psittacine learning and another form of exceptional learning, human second-language acquisition (Pepperberg and Neapolitan 1988).

\section{METHODS FOR EXPERIMENT 1}

\section{SUBJECTS AND HousING}

Subjects were juvenile Grey Parrots, Alo (female) and Kyaaro (male), that were 10 and 6.5 months, respectively, at the beginning of the experiment. They were hand raised and had been obtained from their breeder three months previously. They lived in separate rooms and could not hear one another. Training with live tutors and testing occurred while these birds were atop their cages, on "gyms" (branches that had been nailed together), or on parrot stands. Birds were confined to Hoei cages (ca. $38 \times 71 \times 56 \mathrm{~cm}$ ) when humans were absent, and during sleeping hours. Water and Harrison's Bird Diet were available continuously; fruit, vegetables, dried pastas and cereals were provided when neither testing nor training were in progress.

\section{TRAINING PROCEDURES}

To provide input that varied with respect to social interaction, reference, and contextual applicability, I contrasted sessions of live, videotape, and audiotape 
TABLE 1. Components of different types of tutoring used to train Alo and Kyaaro.

\begin{tabular}{lcll}
\hline \hline & Reference & $\begin{array}{c}\text { Contextual } \\
\text { applica- } \\
\text { bility }\end{array}$ & $\begin{array}{c}\text { Social } \\
\text { inter- } \\
\text { action }\end{array}$ \\
\hline Audiotapes & No & No & No \\
Videotapes & Yes & Partial & No \\
M/R protocol & Yes & Yes & Yes \\
\hline
\end{tabular}

tutoring. Table 1 depicts the variation among the types of tutoring. The birds received the three types of training sessions on a rotating basis, two sessions/ day (morning and afternoon) and five days/week for approximately 11 months beginning 28 June 1991, excluding student vacation periods.

Live tutoring and intrinsic rewards. - The live-tutoring system, called the model/rival (M/R) procedure, was adapted from the work of Todt (1975). M/R training involves three-way interactions between two competent human speakers and the avian student. M/R training primarily introduces new labels and concepts, but also aids in shaping correct pronunciation. Because this study is an in depth comparison of training protocols, I describe the $M / R$ procedure in some detail although the material is available elsewhere (Pepperberg 1981, 1988b, 1990a, b, c).

During $M / R$ training, humans demonstrate to a bird different types of targeted interactions. Sessions begin with a bird observing two humans handling an object; the item is one of several that the bird has previously chosen and used (e.g. as a preening implement; Pepperberg 1981) for three to five consecutive minutes in free-choice sessions over several weeks. One human acts as a trainer, showing the item to the second human, who is both a model for the bird's responses and a rival for the trainer's attention. The trainer queries the model/rival about the item ("What's here?", "What matter?", "What toy?"), giving praise and the object to reward correct answers. The technique thus demonstrates referential and contextual use of labels with respect to observable objects. A trainer shows disapproval for incorrect responses (errors similar to those made by a bird, such as partial identifications, unclear speech) by scolding and temporarily removing the object from sight. Thus, a bird observes aversive consequences of errors. The model/ rival is asked to talk more clearly or try again when a response is incorrect or garbled, thereby allowing the parrot to observe "corrective feedback" (see Goldstein 1984, Vanayan et al. 1985). Because a bird is rewarded for successive approximations to a correct response, the protocol adjusts the level of training to the level of the bird. If a bird is inattentive or its accuracy regresses, trainers threaten to leave ("I' $\mathrm{m}$ gonna go away!'); such behavior is aversive, as our birds become agitated and will beckon ("Come here," "Want tickle") a departing trainer.
Unlike other modeling procedures (e.g. Todt 1975, Goldstein 1984), my protocol requires repeating an interaction while reversing roles of the human trainer and model/rival, and includes the parrot in the interactions. Thus, birds do not simply hear stepwise vocal duets, but observe and learn to engage in a communicative process (i.e. a process that involves reciprocity and can be used by either party to request information or effect environmental change). Without role reversal, birds exhibit two types of behavior that are inconsistent with interactive, referential communication (Todt 1975): (1) they do not transfer responses to anyone other than the human who poses the questions; and (2) they do not learn both parts of the interaction.

The $M / R$ technique specifically demonstrates reference and contextual applicability by using intrinsic reinforcers: Reward for each identification is the item to which the label refers. In contrast, some programs designed to teach communication skills, for both humans and nonhumans, use extrinsic rewards (see Pepperberg 1990a). In these programs, all correct identifications of food or nonfood items or appropriate responses to various specific commands are rewarded with a single item (generally food) that neither directly relates to the skill being taught nor varies with respect to the specific task being targeted. Such extrinsic rewards may delay label acquisition by confounding the label or concept to be learned with some aspect of the reward item (Greenfield 1978, Pepperberg 1981, Miles 1983). My procedure, instead, provides the closest possible association of the label that is being taught and the item to which it refers (Pepperberg 1981).

Videotape presentations.-To provide training that closely followed the $M / R$ procedure but avoided social interaction and minimized contextual applicability, I videotaped the previously trained adult parrot, Alex, during $M / R$ sessions and exposed the juvenile parrots to those tapes. Although Alex already comprehended and used the targeted labels referentially (e.g. Pepperberg 1990a, b), tapes did not present the targeted material as a review session, but followed the lines of actual training. Thus, Alex occasionally erred or interrupted with requests for other objects and changes of location (Pepperberg 1983, 1987a; references to related behavior in other species are Davis 1984, Putney 1985, Moran et al. unpubl. data). As in the live $M / R$ presentations, trainers would also occasionally err. Not only was the style of vocal interaction identical to regular $M / R$ sessions, but tapes also retained patterns of breaks for nonvocal exchanges (e.g. when trainers preened Alex) and timeouts by using, respectively, scenes of such nonvocal interactions or a blank screen. Juveniles watched the videos in isolation, so that no direct social interaction with trainers occurred. By watching a human or Alex produce a particular sound and either receive an object or be scolded, the juveniles saw but did not ex- 
perience directly the effect of a vocalization. Videos, therefore, demonstrated reference but lacked clear contextual applicability.

Audiotape presentations. - Audiotapes were designed not only to parallel the $M / R$ and video procedures, but also to eliminate reference and context. Audiotapes consisted of the audio portion of the video of Alex's sessions on the targeted vocalizations; audio and video sessions, thus, did not differ in amount of auditory input. Juveniles listened to the tapes in isolation and, thus, no objects were associated in any way with the sounds presented over the speaker.

\section{EQUIPMENT}

All tapes were made in the laboratory using an AKG CK8 microphone. Videos were made with a Panasonic SVHS AG-450 camera and TDK HS tapes and were edited with a Panasonic AG1960 proline deck and CT$2082 y$ color monitor. A zoom lens enabled me to include life-size images of Alex and the targeted objects in addition to the somewhat smaller images of the entire training scenario (the object, Alex, and two humans). A previous study (unpubl. data) showed that Alex could accurately label objects presented via a live video link; Grey Parrots thus appear able to recognize two-dimensional video representations. Audiotapes were made and presented on either Sony TCM 5000 or Marantz PMD 221 recorders using Maxell XL-UDII tapes.

\section{LABELS TRAINED aNd CONTROLS FOR SYSTEMATIC BIAS}

Each bird was trained on six labels, two each in the three different conditions. Alo received "cork" and "paper" in M/R sessions, "nail" and "wood" on video, and "key" and "rock" on audiotape. Kyaaro received "paper" and "nail" in M/R sessions, "key" and "cork" on video, and "rock" and "wood" on audiotape. Training sessions were designed to prevent bias that might arise from three sources: (1) differing amounts of exposure to training in each procedure, (2) variable ease of production of labels, or (3) varying motivation to obtain the objects that the labels represented.

Because differing amounts of exposure could influence the efficacy of the various training techniques, I closely matched overall lengths of sessions. Videos and audiotapes were edited into sections that closely approximated natural breaks in live sessions, and overall amounts of training were set to resemble an average of what was given per session to Alex (ca. 30 min per 45-min session), who had received $M / R$ tutoring for over 15 years and had acquired a repertoire of more than 80 referential vocalizations (Pepperberg 1981, 1990a).

Given individual differences between parrots, I could not be sure that each of the chosen labels would be equally easy for each bird to produce. Therefore, I counterbalanced labels, so that, with the exception of "paper" and "rock," labels used for one bird with one technique were used for the other bird with another technique. Both birds were exposed to "paper" via live tutors and to "rock" via audiotape in order to compare their speeds of learning. I chose labels that the aforementioned Alex could clearly produce (Pepperberg 1981, 1990a) to ensure that the vocalizations were within the capacity of the species.

Previous work (Pepperberg et al. 1991) also had shown that motivation affected Alex's label acquisition. In situations in which producing an object label was, at least initially, equivalent to requesting the object, Alex more frequently practiced labels for favored items and acquired these labels more readily. So that motivation would not affect the juveniles' results, labels used in their training referred to items with which they had chosen to interact (see above) in free-play sessions.

\section{Testing Procedures}

To evaluate what the parrots had learned, I administered tests one to four times per week beginning February 1992. The protocol (quoted in part from Pepperberg 1990a) is summarized here. Specific details can be found in Pepperberg (1981).

Criterion prior to testing. - The criterion as to when to begin testing is based on the clarity of a bird's speech, and not on the accuracy of labeling in training (Pepperberg 1981). For testing to begin, a vocalization must be recognized by trainers in blind trials with better than $90 \%$ interobserver agreement. Thus, I separate the effect of our procedures on a bird's ability physically to emit a label from the effect of the procedures on the bird's ability to associate the label and its referent. Only when the former skill is considered satisfactory is the latter skill tested (Pepperberg 1981, 1983).

Precautions against trainer-induced cuing. - Test situations included specific precautions to avoid trainerinduced cuing (Pepperberg 1981). One precaution was a design that prevented either the subject or examiners from predicting which questions (or answers) would appear on a given day. Tests were constructed as follows: The principal trainer (I.M.P.) listed the object labels to be examined. The order of presentation was assigned randomly by a student not engaged in testing. A small number of questions (two to five) was then presented intermittently during training sessions on current (and thus unrelated) topics for several days until all questions were asked. While object labels were being tested, for example, students and I were training sequential number recognition. Training questions ("How many?") were thus as likely during test sessions as a test question ("What's this?"); also, a specific test object might appear only once or twice per session and its appearance could 
not be predicted. A second precaution against cuing was to ensure that trials on a given label were conducted by a student who never trained that label. While training is in progress, students test a number of labels that they do not train, so the presence of a specific student could not cue a bird as to which label would be tested.

Precautions against "expectation cuing",-Intermingling different test questions (e.g. "How many?", "What's this?", "What matter?") during training on other topics also ensures against "expectation cuing" that may occur if a subject "expects" queries on a single topic. Contextual information in single-topic tests could be responsible for a better performance than would otherwise be justified by a subject's actual knowledge of a topic, in that a homogeneous set of questions might lead a subject to ignore all but a small subset of responses. My birds, however, are never queried on a single topic (e.g. object labels) in a session, nor, more importantly, tested successively in one session on similar questions ("What's here?") or on more than three questions that have a particular correct response (e.g. "cork"). Moreover, only novel objects are used for testing and identical exemplars are never used for similar questions (e.g. a bird is asked about shapes, sizes, and colors of trucks, paper and pieces of cork that differ from training exemplars and differ from question to question). A question (with its exemplar) is repeated in a session only if the initial answer is incorrect (see below; Pepperberg 1981). Thus, although the range of correct responses to, for example, "What's here?", "What matter?", or "How many?" was limited initially to just a few object or number labels, in any session a bird had to choose from among several possible responses to the object and number questions to be correct (Pepperberg 1981).

Maintaining the subjects' attention.-Concurrent work on several tasks is also necessary because birds become restless during sessions devoted to a single task. They cease to work, preen, or interrupt with requests for preening (e.g. "Want tickle"). A detailed discussion of such behavior is in Pepperberg (1990a).

Correction procedure. - The number of times objects are presented to a parrot depends upon its accuracy, which is determined as follows: The examiner (a student trainer), presents the targeted object to the bird. The principal trainer sits so that she cannot see the bird, the examiner, or the object being presented. The examiner asks one of the test questions, to which the bird responds. The principal trainer then repeats what she heard the parrot say. This repetition prevents the examiner from accepting an indistinct, incorrect vocalization that is similar to an expected, correct response (e.g. "or" for "cork"). Interpretation of a bird's response is unlikely to be influenced by hearing the type of question. Posttest transcriptions of contextless tapes of Alex's responses agree with the original evaluations to within 98.2\% (Pepperberg 1992b). If what the principal trainer heard is correct (e.g. the appro- priate object label), the bird is given praise and the object and no additional presentations occur (i.e. only a "first trial" response exists). If a response is incorrect or indistinct, the examiner removes the object, turns his/ her head (a brief "time-out"), and says "No!" The examiner then implements a correction procedure in that the misnamed object is immediately (re)presented until a correct identification is made; errors are recorded.

Birds thus find that an incorrect response (e.g. substitution of the label of a more desired item for the one presented) is fruitless; instead, correct responses allow a bird to proceed to a preferred item. Because immediate representation of objects during a test occurs only when response to the initial presentation is incorrect, the protocol penalizes a "win-stay" strategy. Incorrect repetition of a previously correct response (e.g. the name of the previous exemplar) elicits no reward.

Scoring procedure.-Test scores are reported two ways, for "first" and "all trials." First-trial data are the percentage of first trials that are correct and are used for statistical analyses. For comparison, I report the all trials score for each task. The all trials score is the total number of correct identifications (i.e. the predetermined number of tested items) divided by the total number of presentations required. If a bird cannot produce a correct response, I report only first-trial data.

\section{RESULTS OF EXPERIMENT 1}

The data demonstrated that the form of input affected the parrots' acquisition of labels. Neither juvenile acquired utterances after 11 months of exposure to either the audio or the video presentations, although two videotapes of them during these sessions demonstrated that they whistled, squawked, and appeared to attend to the video. Both birds, however, learned vocalizations after exposure to seven months of $M / R$ training, although the two labels that Kyaaro acquired were still too garbled at the end of 11 months to distinguish for testing purposes. After seven additional months of training, these vocalizations could be tested. This additional exposure did not, however, enable Kyaaro to acquire the labels trained in the audio or video conditions.

\section{ALo}

Alo never produced, in the presence of trainers, labels she experienced via video (wood, nail) or audio (key, rock). Tapes of her solitary sound productions also revealed that she did not 
TABLE 2. Results of object-identification tests.

\begin{tabular}{lccc}
\hline \hline Object & Test score & Percent & Erroneous identifications (no, errors) \\
\hline & & & A. Alo \\
Paper & $34 / 40$ & 85 & Cork (4), unintelligible (2) \\
Cork & $34 / 40$ & 85 & Paper (1), unintelligible (5) \\
Wood & $0 / 20$ & 0 & Paper (3), cork (8), four (4), unintelligible (5) \\
Key & $0 / 20$ & 0 & Paper (3), cork (4), four (5), unintelligible (8) \\
Rock & $0 / 20$ & 0 & Paper (1), cork (10), four (2), unintelligible (7) \\
Nail & $0 / 20$ & 0 & Paper (2), cork (4), four (8), unintelligible (6) \\
& & & B. Kyaaro \\
Paper & $34 / 40$ & 85 & Nail (5), unintelligible (1) \\
Cork & $0 / 20$ & 0 & Paper (8), nail (6), unintelligible (6) \\
Wood & $0 / 20$ & 0 & Paper (6), nail (7), unintelligible (7) \\
Key & $0 / 20$ & 0 & Paper (4), nail (13), unintelligible (3) \\
Rock & $0 / 20$ & 0 & Paper (6), nail (9), unintelligible (5) \\
Nail & $35 / 40$ & 87.5 & Paper (1), unintelligible (4)
\end{tabular}

"practice" (see Pepperberg et al. 1991) labels for the targeted objects. On identification tests of these objects beginning in April 1992, Alo scored $0 \%$ on 20 trials for each label (binomial test, $P$ $=0.0261$, chance of $1 / 6)$. Her total score was $0 / 80$. I did not re-present the objects on any trials because she never produced even an approximation to the correct labels.

In contrast, on tests given February through April 1992 on labels taught via the M/R procedure, first trial scores were $34 / 40$ for both cork and paper $(85 \%$, binomial test, $P<0.0001$, chance of $1 / 3$ [possible responses were learned labels or an unintelligible utterance, possibly an attempt at another label]; $P=0.0026$, chance of $1 / 2$ [possible responses of the learned labels only]). Overall scores were $40 / 46(87 \%)$ for cork and for paper. Table 2 gives a breakdown of her errors.

\section{KYAARO}

Kyaaro also did not produce, either in the presence of trainers or in private practice, labels that he experienced via audio (wood, rock) or video (cork, key). He attempted to produce labels taught via the $M / R$ technique (paper, nail) but, at the end of 11 months, ran them together ("ail-er") in a manner too difficult to distinguish by trainers for testing. He did, however, produce clearly differentiated versions of nail and paper during private practice. After several more months of training, his labels were at criterion for testing. Because Kyaaro subsequently had several surgeries, we could not test until 1993. On identification tests for each of these objects beginning the end of May 1993, Kyaaro scored $0 \%$ on 20 trials for each label trained via audio or video tape (binomial test, $P=0.0261$, chance $1 / 6$ ). His total score was $0 / 80$. As before, I did not re-present the objects on any trial. On tests given May through July 1993 for labels taught via the $M / R$ procedure, his first trial scores were $34 / 40$ for paper $(85 \%$, binomial test, $P<0.0001$, chance of $1 / 3 ; P=0.0026$, chance of $1 / 2)$ and $35 / 40$ for nail $(87.5 \%$, binomial test, $P<0.0001$, for chance of $1 / 3$ and $1 / 2$ ).

Interestingly, in the first 11 months, Kyaaro did acquire a few extremely clear vocalizations from informal interactions with trainers: "Hi Kyo," "Want tickle," "Kiss." These utterances are always contextually appropriate; they are used, respectively, when we enter his room but ignore him (e.g. during cleaning or a "time out"), while he bows his head and stretches toward our hands, and when he stretches his beak toward our faces. As Kyaaro always accepts tickles or beak rubs, such utterances cannot be tested and no claims can be made for their referentiality.

\section{DISCUSSION OF EXPERIMENT 1}

The difference in performance of the two juvenile parrots must be examined before the results of the experiments can be interpreted. Although Kyaaro's data eventually matched those of Alo, he required several more months of training. A study of the literature on developmental delays in humans suggested that Kyaaro had many behavior patterns in common with those of "attention-deficit disordered" (ADD) 
children (Barkley 1990): short attention span, inability to wait one's turn, difficulty in focusing on an object, frequent task-irrelevant activity (e.g. self-stimulation, which for Kyaaro is obsessive foot chewing), erratic performance on repetitive tasks, and physical clumsiness. After this study was completed, my students and I found that Kyaaro responded to one of the techniques used for working with ADD children (Sherman pers. comm.). We found that Kyaaro would attend more closely to all training if he received intense physical stimulation (stroking, tickling) for several minutes before a session. Such intervention would not, however, have affected the overall results of this study. In a subsequent series of experiments, Kyaaro learned via the $M / R$ technique and not from other forms of video training even when physical stimulation preceded all types of sessions (unpubl. data).

Thus, the data suggest that referential, contextually applicable, and socially interactive input best facilitates learning. Even Kyaaro's results suggest the effectiveness of social input that contains reference and contextual information. The data do not, however, tell anything about the effect of input that is socially interactive but that lacks reference and contextual applicability.

I attempted to examine the effect of such input as part of a study on numerical competence. While the juvenile parrots were receiving audio, video, and $M / R$ training on object labels, they were also exposed to different numbers of sequential notes on a synthesizer while humans modeled correct numerical responses. Neither bird would attend to training (instead, they would preen, chew their feet, request tickles, etc.) until I used chewable Arabic numerals as rewards. Such behavior suggested, but did not prove, the importance of reference and context for learning. Data from the juveniles' learning, therefore, must be compared with results from a separate experiment in which the adult parrot, Alex, was taught, via the M/R protocol, a nonreferential set of vocalizations that lacked explicit contextual applicability.

\section{METHODS FOR EXPERIMENT 2}

(PART 1)

\section{SUBJECT AND HousING}

The experimental subject, a Grey Parrot named Alex, has been the focus of cognitive and communicative studies since 1977. He had access to all parts of the laboratory (contingent upon his vocal requests; e.g. "Wanna go gym") when trainers were present (8 $\mathrm{h} /$ day); trials thus occurred at various locations. He was confined to a desk top and a wire cage $(62 \times 62$ $\times 73 \mathrm{~cm}$ ) at other times (e.g. sleeping hours). He had no regular access to other parrots, as this study was completed before I acquired the juveniles. Water, a psittacine seed mix, and a limited selection of chewable objects (e.g. wooden plant stakes) were available continuously; fruits, vegetables, whole nuts and other objects (keys, variously shaped pieces of wood, paper, wool, etc.) were provided at his vocal request (e.g. "I want cork.").

When this study began, Alex had been trained exclusively with the $M / R$ procedure and referential rewards. He thereby had learned referential use of labels for 50 different objects, 6 shapes, 7 colors, and quantifiers up to 6 to identify, request, refuse, and categorize objects (Pepperberg 1981, 1983, 1987a). He had been tested on concepts such as the presence or absence of sameness and difference, and on the ability to categorize objects with respect to color, shape, or matter (Pepperberg 1987b, 1988c). Other tests (Pepperberg 1990b) showed that he could comprehend as well as produce all of his color, shape, material, and category labels. He also had functional use of several phrases (e.g. "Come here," "You tickle," "What's that?," "I'm sorry," "You tell me," "Wanna go $X, "$ and "Want $Y$," where $X$ and $Y$ are location and object labels).

\section{Training Procedures}

As part of two other studies, on numerical competence and serial learning, Alex was taught a sequence of eight number labels that had no reference either to specific objects in the laboratory or to previously acquired labels. The set of labels, il ee bam ba oo yuk chil gal, was derived from Korean count labels both to facilitate comparisons with children (Fuson 1988) and to be maximally different from English number labels already in his repertoire. "Bam" (pronounced \baem $\backslash$ ) and "ba" were substituted for the Korean "sam" and "sa" because Alex sometimes had difficulty producing an initial "s." Training occurred four to five times per week, beginning October 1988 and continued until Alex produced a modified form of the sequence in June 1989; we eventually accepted his insistence on $n u k$ in place of $y u k$.

The usual $M / R$ procedure was amended to eliminate as much reference and context as possible. Two humans still engaged in training, but did not emphasize the connection between labels and specific objects or collections. Initially, one human would state "Say number" and the other would produce the string in the absence of any objects. Correct responses were rewarded with vocal praise and the opportunity to request any desired object (Pepperberg 1987a); errors 
were punished by scolding and time-outs. Trainer and model/rival reversed roles periodically.

Alex did not attend to training (e.g. would preen or request toys or many changes of location) until we included a minimal point of reference: a "number board" consisting of a piece of paper with Arabic symbols 1 to 8 traced along the diagonal. The trainer then held the paper in front of the model/rival while asking him/her to "Say number," but did not point to the numbers, refer to them in any manner, or transfer the paper. Training, therefore, lacked the usual context and lacked all but minimal referentiality.

Even with the number board, Alex still often ignored (e.g. turned his back to) the trainers. He would say "No" during his turn or would request various items before replying. Trainers responded to such behavior with a "time-out": They would say "I'm going to go away" and leave the room. They would return if Alex said "Come here," "I'm sorry," or if he attempted the targeted vocalizations. If, after learning part of the sequence, Alex regressed to an earlier version (e.g. omitted a label from the string), he would not be scolded but was asked to 'Say better."

\section{TESTING}

Alex's tests were identical to the juveniles' with respect to precautions against cuing, maintaining his attention, correction procedures, and scoring. The tests differed in only two ways. First, Alex was being tested on more tasks than the juveniles, so his tests included more topics. Second, because the labels did not refer to particular items, correct responses were rewarded with praise and a chance to request a desired object rather than the item to which a label referred.

Initial testing occurred mid-June to mid-December 1989 , excluding student vacation and exam periods. Tests occurred two to four times per week but, given the number of topics covered, trials on Korean labels could occur on average less than once a week. During tests Alex was shown the number board and asked to "Say number."

Alex was also tested on his ability to make $1: 1$ correspondences between the number labels and various quantities of objects (i.e. on his comprehension of the labels). The intention was to see if he would say, for example, "il ee bam" to three items. During such tests, he was shown one to seven objects on a tray and asked to "Say number." Such tests occurred early January to late March 1990.

\section{RESULTS OF EXPERIMENT 2}

(PART 1)

Training on Part 1 took 9 months, which was unusually long (Pepperberg 1981, 1983). Normally, labels containing sounds that are already in Alex's repertoire (e.g. "ee," "oo") are learned
TABLE 3. Condensed journal entries describing order of Alex's acquisition of adapted Korean count labels.

19 Oct. 1988 - Began training on rote sequence of number labels.

8 Nov.-Alex first produced chil in his trainers' presence; he occasionally praised model with "Yeah! That's right" for a correct response during training.

14 Nov.-Clearly vocalized chil and ba independent of all other labels when given the count directive, "Say number."

14-29 Nov.-Gave continuous and more frequent vocalizations of chil ba.

30 Nov.-First vocalization of chil ee bam ba.

30 Nov.-2 Dec.- Tapes of solitary practice showed frequent production of chil, chil ee bam ba, and occasionally il ee bam ba.

2-7 Dec.-Tapes of solitary practice show frequent production of il ee bam ba.

9 Dec.-First vocalized il ee bam ba in a trainer's presence.

19 Dec.-Began to place chil later in list; he frequently produced il ee bam ba (pause) chil.

23 Dec. - Said il ee bam ba chil bail; first evidence of label subsequent to chil.

9-25 Jan. 1989-Tapes during solitary practice showed frequent production of il ee bam bak (pause) chil. The " $\mathrm{k}$ " sound at the end of bak is pronounced.

16 Jan.-First attempt by Alex at a longer, ordered sequence: il ee bam bak ss-uck chil. No evidence for gal.

6-24 Feb.-Often placed a " $k$ " sound on $b a$; also often inserted $s n-u c k$ after $b a$ in presence of trainers. Alex also interrupted with the word chil in its appropriate place when trainer recited complete sequence.

2 Mar.-Continued attempt to recite seven labels: il ee bam ba sn uck chil.

9 Mar.-Alex added an eighth label, although it was not correct: il ee bam ba sn uck chil ee-bail.

20-24 Mar.-First attempt by Alex at vocalizing eight labels of similar phonology and order to training sequence: il ee bam ba oo nuk chil wool. Still no evidence for gal.

24 Apr.-Responded to "Say number" with il ee bam ba oo nuk chil.

9 May-Regressed to il ee bam ba look chil.

10 May-Produced il ee bam ba oo nuk chil, sometimes without oo.

13 June-Produced entire sequence il ee bam ba oo nuk chil gal.

in a few days or overnight (Pepperberg 1983, 1990c, Pepperberg et al. 1991). In this study, however, he acquired these presumably "easy" labels six weeks and five months, respectively, after he produced those that less closely resembled familiar sounds (e.g. "chil"; Table 3).

Alex eventually produced, in order, the string of labels that were modeled, substituting "nuk" 
for "yuk." In 20 tests, he made 4 errors (80\% correct); he omitted "oo" twice, "gal" once, and both "oo" and "gal" once. The probability of obtaining this score by chance was less than 0.0001 (binomial test, chance 0.0002 ). His overall score was $20 / 24(83 \%)$.

Alex could not, however, produce shorter strings when presented with sets of fewer than eight items. His responses were similar to those when asked to "Say number" to sets of eight items. In 10 of 14 trials, he produced the entire string; he omitted " $\mathrm{oo}^{\prime}$ on two trials (during presentations of 1 and 4 items), and omitted "gal" on two trials (during presentations of 3 and 5 items).

\section{METHODS FOR EXPERIMENT 2}

(PART 2)

Alex was again the experimental subject and was housed as in Part 1. After testing on Part 1, students and $I$ began to train Alex, with limited reference and contextuality, on use of the string of labels to refer to quantity. We modeled 1:1 correspondences between the entire string of labels and several different sets of eight objects (e.g. toy cars, pompons). A trainer pointed to each object as the model/rival responded with the relevant number label. We intentionally never modeled quantities less than eight. Training began in late March 1990 and continued for approximately three months, except for student vacation and exam periods.

Alex was tested as in Part 1. Testing began May 1990 and ended August 1990.

\section{RESULTS OF EXPERIMENT 2}

(PART 2)

Even after our modeling of 1:1 correspondences for sets of eight items, Alex failed to respond appropriately to smaller sets. Whatever the quantity on the tray, he most often produced the entire set of labels. Thus, his behavior resembled that of Part 1. In 11 of 14 trials, he produced the entire string; he omitted "oo" on a trial for five items, omitted "ba" on a trial for seven items, and omitted "gal" on a trial for four items.

\section{DISCUSSION OF EXPERIMENT 2}

(PARTS 1 AND 2)

Although the amount of time that Alex needed to acquire these labels was somewhat unusual, his training had never before lacked ref- erence and contextual applicability. His behavior was not necessarily a consequence of his training history, because the juveniles, despite their lack of a similar training history, also failed to attend to socially interactive input that lacked reference and contextual applicability (e.g. the first attempt at training sequential numbers). Lack of these features may, however, have affected Alex's motivation to learn. In previous studies, his desire for the object to which a label referred affected the speed of his acquisition of that label (Pepperberg et al. 1991). Thus, the lack of referential reward and contextuality likely contributed to his lack of interest in sessions and his delay in acquisition.

Alex's failure to comprehend the labels in Part 1 was not surprising. His training intentionally failed to provide either any understanding of the individual meanings of the labels he heard or any clear connection between use of the labels and his environment (i.e. did not provide reference or specific contextual applicability). He had been shown and had learned merely an association between events (i.e. to produce a rote series in response to a patterned sheet of paper and the command "Say number').

Even the demonstration in Part 2 of 1:1 correspondence between the full set of labels and the full set of objects provided conditions that were inadequate for a Grey Parrot to learn to comprehend as well as produce a set of labels. Such training provided only a limited sense of context and no direct information about the meaning of individual labels. Previous data (e.g. Pepperberg and Brezinsky 1991) suggest that, had his training in Part 2 been explicitly referential, he might have succeeded. For example, had Alex been referentially taught the relationship between a few subsets of the labels and the appropriate subsets of objects (e.g. the connection between "il ee" and two keys, and "il ee bam" and three cups), he could have transferred such learning to the remaining subsets. The point of the study, however, was to examine what he would acquire with only limited reference and contextuality.

Two inferences can be made from these findings. First, social interaction can, to a degree, compensate for input with only minimal reference and contextuality, but learning from such input will occur more slowly than from input that is also referential and contextually applicable. Second, input that is socially interactive, 
but that provides only limited reference and contextual applicability, enables a learner to produce but not comprehend the code that is trained.

\section{GeNERAL Discussion}

Social interaction, reference, and full contextual experience are all important factors in learning to produce and comprehend an allospecific code even for a mimic such as the Grey Parrot. Absence of some of these factors affects whether and how learning occurs. The effects of each condition can be described in some detail.

When all three factors are missing, as in audiotape presentations, allospecific vocal learning does not occur. Although the juveniles reacted to the sounds of the tape recorder with parrot noises (e.g. whistles and squawks), they made no attempt to reproduce the human sounds contained on the tape. In a sense, the sounds may have been no more than interesting background noises. The birds were given no opportunity to deduce explicit meanings for the sounds and were not shown the purpose for which the sounds could be used. Their response to the sounds had no effect on what they subsequently heard or received, either vocally or physically. Therefore, they had no reason to acquire the sounds. Even so, they might have learned the sounds from the tapes and then either produced a sound at random or in connection with some irrelevant cue, or made some association between the novel sound and the novel object that, subsequently, was presented to them. They did not, however, behave in any of these ways. According to social-modeling theory (e.g. Bandura 1971, 1977), acquisition of any form of exceptional learning (especially second-language acquisition; see Snow et al. 1976) is unlikely for humans under such conditions, and the same appears true for Grey Parrots.

The presence of reference and limited context in the absence of interaction is also not sufficient for allospecific vocal learning. Although the birds attended to the videos, they did not acquire the sounds that they saw modeled. In the absence of social interaction, the juveniles could have failed to learn for at least three reasons: (1) they failed to realize that the interaction that they observed could be transferred to their own situation; (2) they could not deter- mine exactly what aspect of Alex's behavior was actually causing the transfer of the desired objects; or (3) they simply stopped responding to what they saw on tape because they received no encouragement for what could have been their first approximations to the targeted vocalization (i.e. training was not adjusted to their level of competence). Clearly, merely watching another individual receive objects for producing particular sounds provided insufficient input for learning. These data are consistent with findings that demonstrate that verbal interactions are necessary for a child to learn how and why to use a second language (a form of exceptional learning; Pepperberg and Neapolitan 1988). Children who are exposed to a second language solely through television fail to acquire more than a few words (Snow et al. 1976), even if the shows have a tutorial style (LarsenFreeman 1979).

In the experiment with Alex, the presence of social interaction and severely limited context and reference provided, at best, conditions for production but not comprehension of an allospecific code. In this case, the parrot received positive feedback merely for making a particular sound in response to a specific cue; the bird was given no reason to work towards understanding what it was saying or the appropriateness of the vocalization. If acquired, such a vocalization is unlikely to be generalized to related situations. Such training represents most learning situations of mimetic birds that are pets, and explains why parrots were once thought incapable of doing more than randomly mimicking human speech sounds (e.g. Lenneberg 1973). Note that Alex's data are consistent with those of studies on the use of nonreferential, socially interactive input for other forms of exceptional learning (e.g. with children who are [also] learning a second language). Krashen (1982) has shown that children may fail to acquire (or acquire very slowly) foreign languages spoken in their homes when the input provides few references to the objects or situations to which the child is attending and more references to other aspects of the environment. Similarly, adult humans whose foreign-language companions do not provide reference or demonstrate contextual applicability will likely fail in their attempt to acquire the second language (see Burling 1981, Winitz 1981, Klein 1986, also Pepperberg and Neapolitan 1988).

Most likely, social interaction and context 
without reference will also engender production without comprehension. Such is probably the case for pet birds that learn to produce, for example, "Hello" or "Bye, bye" routines ("Good night dear," "Good-bye, and thank you"; Amsler 1947) appropriately but do not comprehend the use of the individual words in these routines. These birds may have a more general sense of the situations in which their vocalizations can be used than do birds taught without context, but cannot be said to have acquired full functional use of the part of the allospecific code they have acquired.

Based on the predictions of social-modeling theory (Bandura 1977, Pepperberg 1991), I suggest that Grey Parrots are also unlikely to acquire comprehension of elements of an allospecific code from input that is referential, fully contextually applicable, but noninteractive. Thus, the presence or absence of an item that could be considered a reward is likely to be less important than the presence or absence of social interaction. In the $M / R$ training, for example, the presence of a reward is unlikely to be the most critical factor in learning because a bird is rewarded only after it has made an attempt at the targeted label; that is, reward occurs only after some learning has taken place. Moreover, the reward primarily reinforces referentiality. Data on nonvocal allospecific learning in a nonhuman primate provide some corroboration. For example, a chimpanzee (Pan troglodytes), who learned non-interactively to produce symbols based on human language to answer questions or make requests, was unable to generalize to related situations (see Savage-Rumbaugh et al. $1980 a, b)$. Like this chimpanzee, parrots might learn to produce appropriate elements of the code, but comprehension would similarly be lacking. Because it is conceivable (although unlikely) that parrots could learn under conditions unfavorable for chimpanzees, such conditions remain to be tested.

All of these experiments, however, involve teaching a referential allospecific code to subjects in a laboratory situation. Thus, two questions arise as to the general validity of the experiments: (1) To what extent is natural parrot communication referential (i.e. why examine referentiality if parrots do not normally engage in referential communication)? (2) Under what conditions might allospecific learning occur in nature (i.e. how does this study relate to conditions in the real world)?
No information exists concerning Grey Parrots' use of referential vocalizations in the wild (Forshaw 1989). Limited data for other psittacine species, however, suggest that referentiality is a characteristic for which one might fruitfully search. Some parrots have intrapair duets that are distinct from interpair or other interparrot vocalizations; these duets may mediate interactions among flock members (Gwinner and Kneutgen 1962, Mebes 1978, Wickler 1980). Such duets are more complex and take longer to learn than those of passerines (e.g. wrens; Farabaugh 1982). If complex communication developed in response to, and in order to mediate, complex social interactions (Humphrey 1978, Crook 1983, Burling 1985), such vocalizations could be referential. So far, Yamashita (1987) reported cooperative vocal "sentinel" behavior in flocks of Indigo Macaws (Anodorhynchus leari) as part of their extensive social organization, Gnam (1988) suggested that calls are used for individual recognition of mated pairs within groups of Bahama Amazons (Amazona leucocephala bahamensis), and Pepper's data (pers. comm.) suggest individual vocal recognition and contextual calls in Glossy Black Cockatoos (Clyptorhynchus lathami; note Saunders 1983). Moreover, a Grey Parrot would not likely acquire referential communication in the laboratory unless such behavior (e.g. Pepperberg $1990 \mathrm{a}, 1992 \mathrm{~b}$, in press) were based on a preexistent cognitive architecture (Rice 1980, Premack 1983).

Comparing laboratory and fieldwork on Grey Parrots is, however, difficult because no studies exist that describe the conditions under which Grey Parrots acquire or use allospecific vocalizations in nature. A recent paper (Cruickshank et al. 1993) provides the only evidence that such allospecific learning occurs. Conceivably, research such as mine will provide an impetus for the appropriate field studies.

In sum, I have sought to determine the conditions necessary for the acquisition of a referential, allospecific communication code by Grey Parrots. Even though mimetic birds are characterized by their extensive capacities to acquire allospecific vocalizations, Grey Parrots (at least) seem to learn such a code most readily under certain environmental conditions. Although some combinations of conditions remain to be tested for these birds (e.g. reference and full contextual applicability in the absence of social interaction; reference and limited con- 
text with either full or limited interaction; the effectiveness of two- versus three-dimensional referents), input that is fully referential, contextually applicable, and socially interactive ensures that these parrots cannot only produce but also eventually comprehend allospecific vocalizations (Pepperberg 1987a, b, 1990b, 1992b). Lack of some or all of these aspects will affect the course of acquisition and will likely prevent full allospecific learning from occurring.

\section{ACKNOWLEDGMENTS}

This work was supported by grants to I.M.P. from the National Science Foundation (BNS 88-20098 and 91-96066) and the NSF Research Experience for Undergraduates program, and to students in the University of Arizona Undergraduate Biology Research Program. I thank Madonna and Russ LaPell of VIP Aviaries for donation of Alo and Kyaaro, Gloria Dolan of Emerald Bird Caddy for donation of cages, gyms, and stands, and Pam Banta, Mike Brezinsky, Robyn Bright, Sean Garcia, Stacey Humble, Eric Jackson, Jane Lewis, Sharon Marconi, Diana May, Dianne Patterson, Jayme Silverstone, Norma Quevedo, Susan Turney, Audrey Warren, Denice Warren, and Rebecca Zweifel for help as secondary trainers. I thank Pam Banta, Luis Baptista, Donald Kroodsma, Lewis Petrinovich, Meredith West and two unknown referees for comments on an early version of the manuscript.

\section{LITERATURE CITED}

AMSLER, M. 1947. An almost human Grey Parrot. Avicult. Mag. 53:68-69.

BANDURA, A. 1971. Analysis of modeling processes. Pages 1-62 in Psychological modeling (A. Bandura, Ed.). Aldine-Atherton, Chicago.

BANDURA, A. 1977. Social modeling theory. AldineAtherton, Chicago.

BAPTISTA, L. F. 1988. Imitations of White-crowned Sparrow songs by a Song Sparrow. Condor 90: 486-489.

BAPTISTA, L. F., AND M. L. MoRTon. 1981. Interspecific song acquisition by a White-crowned Sparrow. Auk 98:383-385.

Baptista, L. F., and L. Petrinovich. 1984. Social interaction, sensitive phases, and the song template hypothesis in the White-crowned Sparrow. Anim. Behav. 32:172-181.

Baptista, L. F., AND L. Petrinovich. 1986. Song development in the White-crowned Sparrow: Social factors and sex differences. Anim. Behav. 34: 1359-1371.

BARKLEY, R. A. 1990. Attention deficit disorder: A handbook for diagnosis and treatment. Guildford Press, New York.

BURLING, R. 1981. Social constraints on adult lan- guage learning. Pages $279-290$ in Native language and foreign language acquisition $(\mathrm{H}$. Winitz, Ed.). New York Academy of Sciences, New York.

BURLING, R. 1985. The selective advantage of complex language. Ethol. Sociobiol. 7:1-16.

Crook, J. H. 1983. On attributing consciousness to animals. Nature 303:11-14.

Cruickshank, A. J., J.-P. Gautier, and C. Chappuis. 1993. Vocal mimicry in wild African Grey Parrots Psittacus erithacus. Ibis 135:293-299.

Davis, H. 1984. Discrimination of the number three by a raccoon (Procyon lotor). Anim. Learn. Behav. 4:121-124.

Farabaugh, S. M. 1982. The ecological and social significance of duetting. Pages 85-124 in Acoustic communication in birds. Vol. 2, Song learning and its consequences (D. E. Kroodsma and E. H. Miller, Eds.). Academic Press, New York.

FORSHAW, J. M. 1989. The parrots of the world. Lansdown Editions, Sydney.

FusoN, K. 1988. Childlren's counting and concepts of number. Springer-Verlag, New York.

GINSBURG, N. 1963. Conditioned talking in the mynah bird. J. Comp. Physiol. Psychol. 56:1061-1063.

GNAM, R. 1988. Preliminary results on the breeding biology of Bahama Amazon. Parrot Letter 1:2326.

GolDstein, H. 1984. The effects of modeling and corrected practice on generative language and learning of preschool children. J. Speech Hear. Disord. 49:389-398.

Gossette, R. L. 1969. Personal communication to O. H. Mowrer, 1980. Pages 105-106 in Psychology of language and learning (O. H. Mowrer, Ed.). Plenum Press, New York.

GREENFIELD, P. M. 1978. Developmental processes in the language learning of child and chimp. Behav. Brain Sci. 4:573-574.

Grosslight, J. H. AND W. C. Zaynor. 1967. Verbal behavior in the mynah bird. Pages 5-19 in Research in verbal behavior and some neurophysiological implications (K. Salzinger and S. Salzinger, Eds.). Academic Press, New York.

Grosslight, J. H., W. C. Zaynor, AND B. L. Lively. 1964. Speech as a stimulus for differential vocal behavior in the mynah bird (Gracula religiosa). Psychon. Sci. 1:7-8.

GWINNER, E., AND J. KNEUTGEN. 1962. Uber die biologische Bedeutung der 'zweckdienlichen' Anwendung erlernter Laute bei Vogeln. Z. Tierpsychol. 19:692-696.

HUMPHREY, N. 1978. Nature's psychologists. New Sci. 78:900-903.

KLeIN, W. 1986. Second language acquisition. Cambridge Univ. Press, Cambridge.

KRASHEN, S. 1982. Principles and practice in second language acquisition. Pergamon Press, Oxford.

LARSEN-FREEMAN, D. 1979. The importance of input 
in second language acquisition. Pages 87-93 in Pidginization and creolization as language acquisition (R. W. Andersen, Ed.). Newbury House, Rowley, Massachusetts.

LENNEBERG, E. H. 1973. Biological aspects of language. Pages 49-60 in Communication, language, and meaning (G. A. Miller, Ed.). Basic Books, New York.

MARler, P., AND S. Peters. 1977. Selective vocal learning in a sparrow. Science 198:519-521.

Marler, P., AND S. Peters. 1987. A sensitive period for song acquisition in the Song Sparrow, $\mathrm{Mel}$ ospiza melodia: A case of age-limited learning. Ethology 76:89-100.

MEBEs, H. D. 1978. Pair-specific duetting in the Peachfaced Lovebird, Agapornis roseicollis. Naturewissenschaften 65:66-67.

Miles, H. L. 1983. Apes and language. Pages 43-61 in Language in primates ( $\mathrm{J}$. de Luce and $\mathrm{H}$. T. Wilder, Eds.). Springer-Verlag, New York.

MoWrER, O. H. 1952. The autism theory of speech development and some clinical applications. J. Speech Hearing Disord. 17:263-268.

MOWRER, O. H. 1954. A psychologist looks at language. Am. Psychol. 9:660-694.

MoWrer, O. H. 1958. Hearing and speaking: An analysis of language learning. J. Speech Hearing Disord. 23:143-152.

PePPERBERG, I. M. 1981. Functional vocalizations by an African Grey Parrot (Psittacus erithacus). Z. Tierpsychol. 55:139-160.

PEPPERBERG, I. M. 1983. Cognition in the African Grey Parrot: Preliminary evidence for auditory/ vocal comprehension of the class concept. Anim. Learn. Behav. 11:179-185.

PePperberG, I. M. 1985. Social modeling theory: A possible framework for understanding avian vocal learning. Auk 102:854-864.

PePPERBERG, I. M. 1986. Acquisition of anomalous communicatory systems: Implications for studies on interspecies communication. Pages 289-302 in Dolphin behavior and cognition: Comparative and ecological aspects ( $R$. Schusterman, J. Thomas, and F. Wood, Eds.). Erlbaum Associates, Hillsdale, New Jersey.

PePperberG, I. M. 1987a. Evidence for conceptual quantitative abilities in the African Grey Parrot: Labeling of cardinal sets. Ethology 75:37-61.

PepperberG, I. M. 1987b. Acquisition of the same/ different concept by an African Grey Parrot (Psittacus erithacus): Learning with respect to color, shape, and material. Anim. Learn. Behav. 15:423432.

Pepperberg, I. M. 1988a. The importance of social interaction and observation in the acquisition of communicative competence: Possible parallels between avian and human learning. Pages $279-$ 299 in Social learning: Psychological and biolog- ical perspectives (T. R. Zentall and B. G. Galef, Eds.). Erlbaum Associates, Hillsdale, New Jersey.

PEPPERBERG, I. M. 1988b. An interactive modeling technique for acquisition of communication skills: Separation of "labeling" and "requesting" in a psittacine subject. Appl. Psycholinguist. 9:59-76.

PEPPERBERG, I. M. 1988c. Comprehension of "absence" by an African Grey Parrot: Learning with respect to questions of same/different. J. Exp. Anal. Behav. 50:553-564.

PepPERBerg, I. M. 1990a. Some cognitive capacities of an African Grey Parrot (Psittacus erithacus). Pages 357-409 in Advances in the study of behavior, vol. 19 (P. J. B. Slater, J. S. Rosenblatt, and C. Beer, Eds.). Academic Press, New York.

PePperberg, I. M. 1990b. Cognition in an African Grey Parrot (Psittacus erithacus): Further evidence for comprehension of categories and labels. J. Comp. Psychol. 104:41-52.

PEPPERBERG, I. M. 1990c. Referential mapping: A technique for attaching functional significance to the innovative utterances of an African Grey Parrot (Psittacus erithacus). Appl. Psycholinguist. 11:23-44.

PePperberg, I. M. 1991. Learning to communicate: The effects of social interaction. Pages 119-164 in Perspectives in ethology, vol. 9 (P. P. G. Bateson and P. H. Klopfer, Eds.). Plenum Press, New York.

Pepperberg, I. M. 1992a. What studies on song learning can teach us about playback experiments. Pages 47-57 in Playback and animal communication: Problems and prospects (P. K. McGregor, Ed.). Plenum Press, New York.

PePPERBERG, I. M. 1992b. Proficient performance of a conjunctive, recursive task by an African Grey Parrot (Psittacus erithacus). J. Comp. Psychol. 106: 295-305.

PEPPERBERG, I. M. 1993. A review of the effects of social interaction on vocal learning in African Grey Parrots (Psittacus erithacus). Neth. J. Zool. 43:104-124.

PePPERBERG, I. M. 1994. Numerical competence in an African Grey Parrot. J. Comp. Psychol. 108:1-9.

PepPerberg, I. M., K. J. Brese, AND B. J. HARris. 1991. Solitary sound play during acquisition of English vocalizations by an African Grey Parrot (Psittacus erithacus): Possible parallels with children's monologue speech. Appl. Psycholinguist. 12:151178.

PePPerberg, I. M., AND M. V. BREZINSKY. 1991. ACquisition of a relative class concept by an African Grey Parrot (Psittacus erithacus): Discriminations based on relative size. J. Comp. Psychol. 105:286294.

PePperberg, I. M., AND D. M. Neapolitan. 1988. Second language acquisition: A framework for studying the importance of input and interaction in exceptional song acquisition. Ethology 77:150168 
PEPPERBERG, I. M., AND L. SCHINKE-LLANO. 1991. Language acquisition and form in a bilingual environment: A framework for studying birdsong in zones of sympatry. Z. Tierpsychol. 89:1-28.

PremaCK, D. 1983. The codes of man and beast. Behav. Brain Sci. 6:125-167.

Putney, R. T. 1985. Do willful apes know what they are aiming at? Psychol. Record 35:49-62.

RICE, M. 1980. Cognition to language: Categories, word meanings, and training. University Park Press, Baltimore.

SAUNDERS, D. A. 1983. Vocal repertoire and individual vocal recognition in the short-billed Whitetailed Black Cockatoo, Calyptorhynchus funereus latirostris Carnaby. Aust. Wildl. Res. 10:527-536.

Savage-Rumbaugh, E. S., D. M. Rumbaugh, aND S. BoYsEN. 1980a. Do apes use language? Am. Sci. 68:49-61.

SAVAGE-RuMbaUGH, E. S., D. M. RuMbaUgh, S. T. SMITH, AND J. LAwSON. 1980b. Reference: The linguistic essential. Science 210:922-925.

SLATER, P. I. B. 1991. Learned song variations in British storm-petrels? Wilson Bull. 103:515-517.

SNOW, C. E, A. ARLMAN-Rupp, Y. HASSING, J. JobSE, J. JOOSTEN, AND J. VORSTER. 1976. Mother's speech in three social classes. J. Psycholinguist. Res. 5:120.

ToDT, D. 1975. Social learning of vocal patterns and modes of their applications in Grey Parrots. Z. Tierpsychol. 39:178-188.

VANAYAN, M., H. A. ROBERTSON, AND G. B. BIEDERMAN. 1985. Observational learning in pigeons: The effects of model proficiency on observer performance. J. Gen. Psychol. 112:349-357.

West, M. J., A. N. Stroud, AND A. P. KING. 1983. Mimicry of human voice by European Starlings: The role of social interaction. Wilson Bull. 95: 635-660.

WICKLER, W. 1980. Vocal duetting and the pairbond. I. Coyness and the partner commitment. Z. Tierpsychol. 52:201-209.

WINITZ, H. 1981. Input consideration in the comprehension of first and second languages. Pages 296-308 in Native language and foreign language acquisition (H. Winitz, Ed.). New York Academy of Sciences, New York.

YAMASHITA, C. 1987. Field observations and comments on the Indigo Macaw (Anodorhynchus leari), a highly endangered species from northeastern Brazil. Wilson Bull. 99:280-282. 